\title{
Apply Awaji-shima Consensus Conference Criteria Before Diagnosing Amyotrophic Lateral Sclerosis
}

\author{
Josef Finsterer $^{*, 1}$ and Claudia Stöllberger ${ }^{2}$ \\ ${ }^{1}$ Danube University Krems, Krems, Krankenanstalt Rudolfstiftung, Vienna, Austria \\ ${ }_{2}^{2}$ Medical Department, KrankenanstaltRudolfstiftung, Vienna, Austria
}

\begin{abstract}
Objectives: ALS may be diagnosed although affection of other organs suggests another pathogenetic background.

Case report: In a 72yo non-smoking male progressive gait disturbance with recurrent falls since $2 \mathrm{y}$ was initially attributed to axonal polyneuropathy. Additionally, he had arterial hypertension, diabetes, hyperlipidemia, hyperuricemia, hyper-CKemia, hepatopathy, atrial fibrillation, recurrent heart-failure, pulmonary hypertension, mitral insufficiency, and restrictive cardiomyopathy. Possible causes of polyneuropathy were diabetes, long-standing alcoholism, folate-deficiency, or hereditary disease. Later the patient was re-diagnosed as ALS despite absence of upper motor-neuron or bulbar signs, the presence of multiple risk factors for polyneuropathy, of stocking-type sensory disturbances, and of cardiac abnormalities, which could explain dyspnea. Misdiagnosing polyneuropathy as ALS stigmatized the patient and prevented him from further diagnostic work-up for cardiac disease and adequate treatment for heart-failure. Though the diagnosis of ALS was withdrawn, he was put on comfort care and opiates were given when dyspnea acutely deteriorated to death without further cardiac or pulmonary investigations or specific cardiac treatment.
\end{abstract}

Conclusions: ALS should be diagnosed only if the Awaji-shima criteria are fulfilled and if all differential diagnoses were profoundly excluded. Respiratory insufficiency should not be attributed to bulbar involvement in ALS as long as cardiac, pulmonary, or myopathic causes were excluded.

Keywords: Motor neuron disease, anterior horn cell disease, amyotrophic lateral sclerosis, mimicry, polyneuropathy, cardiomyopathy, cardiac involvement.

\section{INTRODUCTION}

Though criteria for diagnosing amyotrophic lateral sclerosis (ALS) were repeatedly modified and adapted according to the needs of clinicians, diagnosing ALS is still challenging [1-3]. This is because diagnosing ALS relies on progressive abnormalities on clinical and electrophysiological investigations and the absence of differentials that could explain the clinical presentation. There is no single biomarker or other laboratory parameter, which would allow a quick and reliable diagnosis even in the early stages of the disease. The diagnosis is even more challenging if neurological and nonneurological abnormalities are present, which are usually not associated with ALS and do not fit to the definition of ALS, as in the following case.

\section{CASE REPORT}

A 72 yo non-smoking Caucasian male, height $170 \mathrm{~cm}$, weight $79 \mathrm{~kg}$, was admitted for dyspnea with respiratory acidosis. He had a 7y-history of diabetes and a $2 y$ history of progressive gait disturbance with recurrent falls complicated

*Address correspondence to this author at the Postfach 201180 Vienna, Austria, Europe; Tel: +43-1-71165-92085; Fax: +43-1-4781711;

E-mail: fifigs1@yahoo.de by rib fractures $1 \mathrm{y}$ ago, a subdural hematoma $6 \mathrm{~m}$ earlier, and a right-sided shoulder trauma. Until $2 \mathrm{~m}$ before admission he was able to walk with a walker and was using a wheel chair since then. He had had a massive alcohol problem until 3y earlier. Additionally, the history was positive for transient bilateral amaurosis $2 y$ earlier. Diagnostic work-up following a syncope after application of nitroglycerine $18 \mathrm{~m}$ ago, revealed arterial hypertension, atrial fibrillation, bradycardia as low as 40beats/min, myocardial thickening, mitral insufficiency, hyperlipidemia, hyperuricemia, hepatopathy, and hyper-CK-emia up to 409U/l (n, <171U/l). Cerebral CT and MRI showed a right-sided thalamic lacuna, leucaraiosis, and non-specific areal white matter lesions. He scored 24 of 30 points on the mini-mental state examination. Oral anticoagulation was waived because of the falls. Since then he developed cold feet and paresthesias of the distal lower limbs. The family history was positive for diabetes (daughter, sister) and dementia (brother).

Diagnostic work-up for heart failure $12 \mathrm{~m}$ prior to admission revealed leg edema, persistent atrial fibrillation, pulmonary hypertension, slight mitral and aortic valve insufficiency, enlargement of both atria, small pericardial effusion, and folic acid deficiency. Nerve conduction studies revealed axonal polyneuropathy and cerebral CT showed bilateral basal ganglia and right thalamic lacunas and white matter lesions. Lung disease and peripheral arteriopathy were ex- 
cluded. Neurological investigations $4 \mathrm{~m}$ prior to admission revealed progression of weakness and wasting, microangiopathy on cerebral CT, but absence of upper motor-neuron signs, bulbar signs, GM1 or GQ1b antibodies, and CSF, videocinematography, and pulmonary abnormalities. Nerve conduction studies revealed severe axonal degeneration. Needle electromyography revealed fibrillations, fasciculations, denervation, re-innervation but no giant potentials in three muscles. The Schallong-test was slightly abnormal. Despite these non-typical findings the patient was diagnosed as having ALS and riluzole was begun.

On admission the patient was awake and talkative but compromised by dyspnoe. Except for proBNP elevated to $1759 \mathrm{pg} / \mathrm{nl}$ (n, <278pg/nl) all other serum parameters including HbA1c, renal and liver function parameters, electrolytes, folic acid, vitamin B12, creatine-kinase, and serum lipids were normal. Blood gas analysis showed respiratory acidosis with a maximal pCO2 value of $78 \mathrm{mmHg}$. ECG showed atrial fibrillation. Transthoracic echocardiography revealed normal systolic function, enlarged atria, restrictive filling pattern, and myocardial thickening. Clinical neurologic examination one day after admission revealed left-sided hypoacusis (previous tympanic rupture), temporal disorientation, diffuse weakness of the left arm and distal weakness of the right arm (M4+ to M4-), bilateral weakness with distal predominance of the lower limbs (M4+ to M3-), reduced biceps, triceps and patella tendon reflexes with left-sided predominance, absent Achilles tendon reflexes, fasciculations of the intrinsic hand muscles, stocking-type hypaesthesia and dysaesthesia bilaterally, and gynecomastia. There were neither bulbar nor pyramidal signs. On the third hospital day the patient awaked at night with lung edema but recovered under nitroglycerin, diuretics and morphium. On the next morning he could not be contacted anymore and had severe dyspnea again. A neurologist from outside the hospital recommended "comfort care" why cardiac treatment was discontinued without further examinations and morphium subcutaneously was begun. The patient died a few hours later.

\section{DISCUSSION}

According to the revised El Escorial [2], the Airlie-House criteria,[3] and the Awaji-shima criteria [1], the diagnosis of ALS requires the presence of upper and lower motor-neuron affection, progression of these abnormalities on clinical, electrophysiologic, or neuropathologic examination and absence of neuroimaging, electrophysiologic, or pathologic evidence of other disease processes that might explain upper or lower motor-neuron degeneration (Table 1). The presented patient, initially diagnosed as axonal polyneuropathy, did not fulfill the Awaji-shima criteria because of absent upper motor-neuron signs, stocking-type sensory disturbances, and causes other than ALS that could explain respiratory insufficiency. Though he did not fulfill the Awajishima criteria and though treating physicians themselves expressed some doubt about their diagnosis, he was misdiagnosed as ALS on his second neurological presentation and thus received an etiquette, which strongly influenced his outcome.

Arguments for axonal sensorimotor polyneuropathy in the presented patient are the history (long-term alcohol abuse, diabetes, folate deficiency), the clinical examination (quadruparesis with distal predominance), reduced/absent tendon reflexes, fasciculations, stocking-type sensory disturbances), and the instrumental findings (axonal lesion on nerve conduction studies, neurogenic electromyography). It cannot be definitively excluded, however, that polyneuropathy had a hereditary component since he was abstinent from

Table 1. Awaji-shima Consensus Recommendations for the Application of Electrophysiological Tests to Diagnose ALS, as Applied to the Revised El Escorial Criteria (Airlie House 1998) [1]
1. Principles (from the Airlie House criteria)
The diagnosis of ALS requires
(A) the presence of
(1) evidence of lower motor neuron (LMN) degeneration by clinical, electrophysiological or neuropathological examination
(2) evidence of upper motor neuron (UMN) degeneration by clinical examination; and
(3) progressive spread of symptoms or signs within a region or to other regions, as determined by history, physical examination, or electro- physiological tests

(B) the absence of

(1) electrophysiological or pathological evidence of other disease processes that might explain the signs of LMN and/or UMN degeneration, and

(2) neuroimaging evidence of other disease processes that might explain the observed clinical and electrophysiological signs

2. Diagnostic categories

Definite ALS

defined by clinical or electrophysiological evidence by the presence of LMN as well as UMN signs in the bulbar region and at least two spinal regions or the presence of LMN and UMN

signs in three spinal regions

Probable ALS

defined on clinical or electrophysiological evidence by LMN and UMN signs in at least two regions with some UMN signs necessarily rostral to (above) the LMN signs

Possible ALS

defined when clinical or electrophysiological signs of UMN and LMN dysfunction are found in only one region; or UMN signs are found alone in two or more regions; or LMN signs are found rostral to UMN signs.

Neuroimaging and clinical laboratory studies will have been performed and other diagnoses must have been excluded 
alcohol since 3y, and since folate levels and diabetes were well-controlled on his final admission. At least diabetes and cognitive impairment could be hereditary but it cannot be excluded that the patient suffered from a genetic multisystem disorder. It also cannot be excluded that there was a muscle disease (recurrent CK- elevation), which predominantly affected the respiratory muscles and remained undetected due to the dominance of polyneuropathy. Since fasciculations are not unique to ALS, they neither exclude polyneuropathy nor myopathy [4-6].

Dyspnea in the presented patient can be attributed to heart failure or undetected myopathy. Possible causes of heart failure were restricted cardiomyopathy, atrial fibrillation, pulmonary hypertension, pulmonary embolism, heart valve abnormalities, or arterial hypertension. Coronary artery disease, which has not been excluded by coronary angiography in this patient, could be another explanation [7] but was rather unlikely given the well-controlled diabetes, hyperlipidemia, and blood pressure. Additionally, MR-angiography of the cerebral vessels did not show significant atherosclerosis and carotid ultrasound and ultrasound of the lower limb arteries were normal. A further argument for dyspnea due to heart failure is the beneficial effect of antidiuretic treatment in the night before decease. Hypercapnia with normal oxygen saturation not only reflects muscular respiratory insufficiency but may also occur with heart failure itself [8]. Particularly, high output heart failure, which is a feature of restrictive cardiomyopathy [9], may go along with hypercapnia [10]. Additionally, hypercapnia may be explained with pulmonary hypertension [11].

This case shows that ALS should be diagnosed only if the Awaji-shima criteria are fulfilled and if all differentials were profoundly excluded. Respiratory insufficiency should not be attributed to bulbar involvement in ALS as long as cardiac, pulmonary, or myopathic causes were excluded.

\section{CONFLICT OF INTEREST}

The authors confirm that this article content has no conflicts of interest.

\section{ACKNOWLEDGEMENT}

None declared.

\section{REFERENCES}

[1] Carvalho MD, Swash M. Awaji diagnostic algorithm increases sensitivity of El Escorial criteria for ALS diagnosis. Amyotroph Lateral Scler 2009;10: 53-7.

[2] Brooks BR. El Escorial World Federation of Neurology criteria for the diagnosis of amyotrophic lateral sclerosis. Subcommittee on Motor Neuron Diseases/Amyotrophic Lateral Sclerosis of the World Federation of Neurology Research Group on Neuromuscular Diseases and the El Escorial "Clinical limits of amyotrophic lateral sclerosis" workshop contributors. J Neurol Sci 1994;124(Suppl): 96-107.

[3] World Federation of Neurology Research Group on Neuromuscular Diseases Subcommittee on Motor Neuron Disease. Airlie House guidelines. Therapeutic trials in amyotrophic lateral sclerosis. Airlie House "Therapeutic Trials in ALS" Workshop Contributors. J Neurol Sci 1995;129 (Suppl): 1-10.

[4] Contégal F, Bidot S, Thauvin C, et al. Finnish amyloid polyneuropathy in a French patient. Rev Neurol (Paris) 2006;162: 997-1001.

[5] Nance CS, Klein CJ, Banikazemi M, et al. Later-onset Fabry disease: an adult variant presenting with the cramp-fasciculation syndrome. Arch Neurol 2006;63: 453-7.

[6] Nohira O, Imai N, Okabe T, Hamaguchi K. A Japanese pedigree with oculopharyngeal muscular dystrophy. Rinsho Shinkeigaku 1994;34: 461-5.

[7] Storey RF, Bliden KP, Patil SB, et al. ONSET/OFFSET Investigators. Incidence of dyspnea and assessment of cardiac and pulmonary function in patients with stable coronary artery disease receiving ticagrelor, clopidogrel, or placebo in the ONSET/OFFSET study. J Am Coll Cardiol 2010;56: 185-93.

[8] Passino C, Giannoni A, Milli M, Polettii R, Emdin M. Recent knowledges on chemosensitivity to hypoxia and hypercapnia in cardiovascular disease. Recenti Prog Med 2010;101: 308-13.

[9] Pinamonti B, di Lenarda A, Sinagra G. Heart failure due to diastolic dysfunction: the treatment principles. Ital Heart J Suppl 2000;1: 469-80.

[10] Mehta PA, Dubrey SW. High output heart failure. QJM 2009;102: 235-41.

[11] Forfia PR. Approach to patients with heart failure and pulmonary hypertension. Curr Treat Options Cardiovasc Med 2007;9: 302-9.

Received: February 04, 2012 